\title{
$49 / 1$
}

\section{Cabiers $d u$ MONDE RUSSE}

Janvier-mars 2008

Marc Raeff (1923-2008)

Alain BESANÇON : Hommage à Marc Raeff

Paul BUSHKOVITCH : In memoriam

Articles

John LEDONNE : Russia's eastern theater, 1650-1850, springboard or strategic backyard?

Cécile PICHON-BONIN : Peindre et vivre en URSS dans les années 1920-1930 : commandes, engagements sous contrat et missions de création

Dossier : Réformes et société en Asie centrale tsariste et soviétique

Avant-propos par Isabelle Ohayon

Paolo SARTORI : Judicial elections as a colonial reform: The Qadis and Biys in Tashkent, 1868-1883

Niccolò PIANCIOLA : Décoloniser l'Asie centrale ? Bolcheviks et colons au Semireč'e (1920-1922)

Isabelle OHAYON : Lignages et pouvoirs locaux : l'indigénisation au Kirghizstan soviétique (années 1920-1930)

Benjamin H. LORING : Rural dynamics and peasant resistance in Southern Kyrgyzstan, 1929-1930

\section{Résumés/Abstracts}

Livres reçus

Rédaction :

54, bd Raspail, F-75006 Paris, cmr@ehess.fr, http://monderusse.revues.org

Vente au numéro:

CID, 131 bd Saint-Michel, F-75005 Paris, cid@msh-paris.fr

Abonnements :

service des abonnements EHESS, bât. Copernic, 20, avenue Édouard Herriot, 92350 Le Pessis-Robinson 


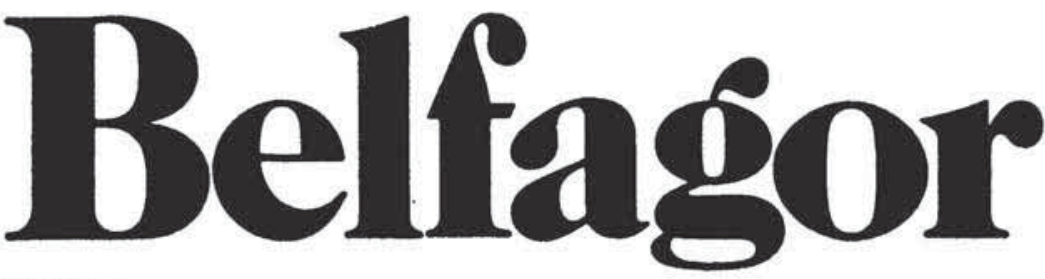

rassegna

di varia umanità

ANNO LXIV • FASCICOLO 2 • 31 MARZO 2009

fondata da

Luigi Russo

diretta da

Carlo Ferdinando Russo

RITRATTI CRITICI DI CONTEMPORANEI

GIOVANNi Morelur: Luciano Berio

Giuseppe Guido Pullía: Franco Basaglia

DIREZIONE

Prof. C.F. Russo

Casella postale 291 - 70100 Bari

Tel. e fax 080.55.41. 534

EMAIL:

cf.russo@lgxserve.ciseca.uniba.it

adele.plotkin@libero.it

REDAZIONE

Mario Isnenghi condirettore

Raffaele Ruggiero segretario

Franco Arato

Emanuele Cutinelli-Rendina

Ugo Dotti

Pasquale Guaragnella

Vitilio Masiello

Antonio Resta

Adele Russo

Onofrio Vox

\section{ABBONAMENTO ANNUO}

2009 (6 FASCICOLI)

ITALIA: Istituzioni* $€ 64,00$

Privati $€ 49,00$

ForeigN: Institutions $€ 112,00$

Individuals $€ 86,00$

*La quota per le istituzioni è comprensiva dell'accesso online alle riviste

* Prices for institutions indude access

to the online version of the joumals

sostenitore $\quad € 200,00$

un fascicolo $\quad € 23,00$

onc issuc, foreign $€ 27,00$

fascicoli arretrati $€ \in 28,00$

annate arretrate 21020509 *Belfagor", Firenze

http://belfagor.olschki.it

SAGGI E STUDI

DAvide Colussr: Spitzer e la pianticella di Croce

Franco Arato: I Gesuiti spagnoli nell'Italia del Settecento

\section{VARIETÀ E DOCUMENTI}

LIDIA DE FEDERICIS: Romanzo di gente che scrive

Sandro Gerbi: Max Ascoli, Nelson Rockfeller e il Perú di Antonello Gerbi (1941-1943)

Enrico Trozzo: Penne nostrane nel cielo di Stociolma, 1958

NOTERELLE E SCHERMAGLIE

Renzo Villa: L'chayim, tovaris Stalin!

Nullo Minissi: Il tirocinio di Mariastella

Simona Micali: La cittadella degli esordienti

\section{RECENSIONI}

Orsana Robski, Nessun rimorso (Galina Denissova)

Viktor Erofeev, Il buon Stalin (RenzoVilla)

Francesco Panigarola, Vita scritta da lui medesimo, edizione critica a cura di Fabio Giunta (Francesco Ferretti)

LIBRI RICEVUTI postillati: Battisti Bertini

Brilli Cassio Martin Petrarca Pizzamiglio

Stead-Védrine Stegagno Picchio Carlo Dionisotti

Giorgio Melchiori Sandro Barbera

Una sestina di Picasso

Nec mora 

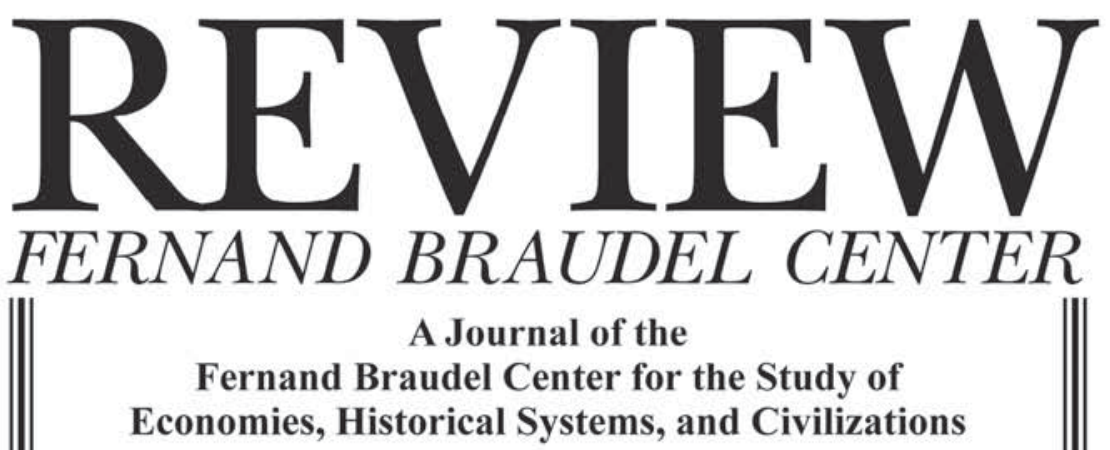

Vol. XXXII includes two special issues: World Food Crisis edited by Philip D. McMichael and Immanuel Wallerstein's new translation of Fernand Braudel, "Histoire et sciences sociales: La Longue durée" (1958) with his introduction to its making.

Previous Special Issues and Sections still available include:

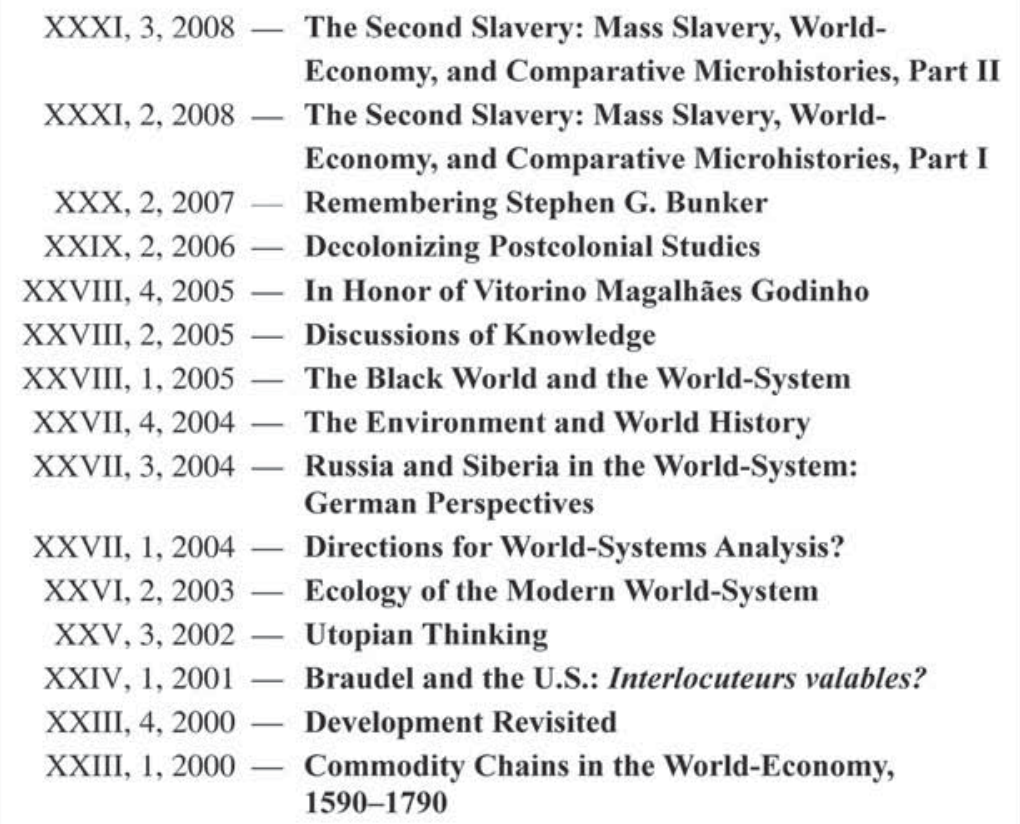

A brochure containing the Table of Contents of past issues is available on request.

Institutions $\$ 125 / \mathrm{yr}$.

Individuals $\$ 28 / \mathrm{yr}$.

Non-U.S. addresses,

postage $\$ 12 / y r$.

Special rate for low gnp

per capita countries $\$ 10 / y r$.

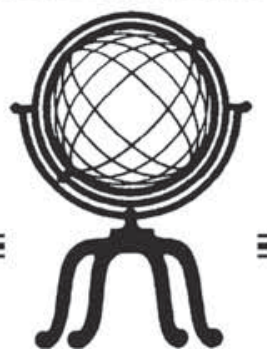

Managing Editor, Review Fernand Braudel Center Binghamton University State University of New York PO Box 6000 Binghamton, NY 13902-6000 


\section{Instructions aux auteurs}

Les Annales n'acceptent que les travaux originaux et inédits. Tout manuscrit proposé est soumis au comité de direction.

Les manuscrits doivent être envoyés à la rédaction en quatre exemplaires accompagnés d'un fichier informatique composé avec un logiciel de type Word. Le texte doit être présenté en double interligne.

La longueur des textes ne doit pas dépasser 90000 signes (notes et espaces compris). Cartes ou graphiques doivent être remis en format natif, PDF ou EPS.

Tout manuscrit doit être accompagné d'un résumé en français et en anglais, d'environ 800 signes, précédé de son titre.

Les articles et comptes rendus non retenus ne sont ni renvoyés ni conservés. 
Abonnement 2009 / Subscription 2009

$\square$ Je souhaite souscrire un abonnement d'un an (6 numéros) à / I want to subscribe for one year (6 issues) to :

\section{Annales. Histoire, Sciences sociales}

\begin{tabular}{lcc} 
& FRANCE / DOM-TOM & ÉTRANGER \\
\multicolumn{1}{c}{ Tarifs } & $\mathbf{1}$ an $\left(\mathbf{6} \mathbf{~ n}^{\mathrm{os}}\right)$ & $\mathbf{1}$ an $\left(\mathbf{6} \mathbf{n}^{\mathrm{os}}\right)$ \\
Particuliers / Individuals & $\square \quad 81 €$ & $\square 104 €$ \\
Institutions / Institutions & $\square 119 €$ & $\square 149 €$ \\
Étudiants / Students* & $\square \quad 62 €$ &
\end{tabular}

* Sur présentation de la carte / Student card required

Nom / Name Prénom / First name

Adresse / Addres

Code postal / Postal code

Ville / Town

Pays / Country

Choisissez votre mode de paiement / Please check how you wish to pay

$\square$ Directement, par chèque, Eurochèque / Directly, by cheque, Eurocheque :

$\square$ Par carte de crédit / By credit card : $\square \mathrm{CB} \quad \square$ Visa $\square$ Master Card / Eurocard

Carte $\mathrm{N}^{\circ} /$ Card $N^{\circ} \mid$

$\square$ Envoyez-moi un reçu / Please, send me a receipt $\quad$ Signature :

Adressez votre commande et votre paiement à l'ordre de / Send your order and payment to

COLIN-ABONNEMENTS - 5 rue Laromiguière, 75240 Paris Cedex 05

Date de validité de l'offre : 31/12/2009

— Les abonnements sont mis en service dans un délai maximum de quatre semaines après réception de la commande et du règlement / Subscriptions begin 4 weeks following receipt of payment.

- Les abonnements partent du premier numéro de l'année (parution début mars) / Subscriptions begin with the first issue of calendar year (March).

— Les réclamations pour les numéros non reçus doivent parvenir dans un délai maximum de six mois / Claims may be submitted to the publisher for missing issues for a period of six months after publication of each individual issue.

- Numéros séparés de l'année et volumes antérieurs / Back issues and volumes (jusqu'à épuisement du stock) :

Revues Armand Colin - 5, rue Laromiguière 75240 Paris cedex 05

Tél. : Numéro Indigo : 0820065095 (France) - 33140464989 (Étranger) - Courriel : infos@armand-colin.fr 
Tous droits de traduction, d'adaptation et de reproduction par tous procédés, réservés pour tous pays. Le Code de la propriété intellectuelle n'autorisant, aux termes de l'article L. $122-5,2^{\circ}$ et $3^{\circ}$ a, d'une part que les « copies ou reproductions strictement réservées à l'usage privé du copiste et non destinées à une utilisation collective » et, d'autre part, que les analyses et les courtes citations dans un but d'exemple et d'illustration, «toute représentation ou reproduction intégrale ou partielle faite sans le consentement de l'auteur ou de ses ayants droit ou ayants cause est illicite » (art. L. 122-4 du CPI). Cette représentation ou reproduction, par quelque procédé que ce soit, constituerait donc une contrefaçon sanctionnée par les articles L. 335-2 et suivants du Code de la propriété intellectuelle.

Diffusion Armand Colin, Paris — Dépôt légal : 2009 — No 3, mai-juin 2009

Imprimé par l'Imprimerie Chirat, 42540 Saint-Just-la-Pendue, nº 5950 ISSN 0395-2649

Imprimé en France 